\title{
After-hours colorectal surgery: a risk factor for anastomotic leakage
}

\author{
Niels Komen • Jan-Willem Dijk • Zarina Lalmahomed • \\ Karel Klop • Wim Hop • Gert-Jan Kleinrensink • \\ Hans Jeekel • W. Ruud Schouten • Johan F. Lange
}

Accepted: 26 February 2009 / Published online: 21 March 2009

(C) The Author(s) 2009. This article is published with open access at Springerlink.com

\begin{abstract}
Purpose This study aims to increase knowledge of colorectal anastomotic leakage by performing an incidence study and risk factor analysis with new potential risk factors in a Dutch tertiary referral center.

Methods All patients whom received a primary colorectal anastomosis between 1997 and 2007 were selected by means of operation codes. Patient records were studied for population description and risk factor analysis.

Results In total 739 patients were included. Anastomotic leakage (AL) occurred in $64(8.7 \%)$ patients of whom nine $(14.1 \%)$ died. Median interval between operation and
\end{abstract}

This work was presented at the Dutch Surgical Days, May 22nd 2008, Veldhoven, the Netherlands.

\section{N. Komen $(\bowtie)$}

Department of Surgery, ZNA Middelheim,

Lindendreef 1 ,

2020 Antwerp, Belgium

e-mail: nielskomen@hotmail.com

N. Komen · J.-W. Dijk • Z. Lalmahomed $\cdot$ K. Klop $\cdot$ H. Jeekel W. Ruud Schouten · J. F. Lange

Department of Surgery, University Medical Center Rotterdam, Erasmus MC, Dr. Molewaterplein 50,

3015 GE Rotterdam, The Netherlands

\section{G.-J. Kleinrensink}

Department of Neurosciences and Anatomy,

University Medical Center Rotterdam,

Erasmus MC, Dr. Molewaterplein 50,

3015 GE Rotterdam, The Netherlands

W. Hop

Department of Epidemiology and Biostatistics,

University Medical Center Rotterdam,

Erasmus MC, Dr. Molewaterplein 50,

3015 GE Rotterdam, The Netherlands diagnosis was 8 days. The risk for AL was higher as the anastomoses were constructed more distally $(p=0.019)$. Univariate analysis showed duration of surgery $(p=0.038)$, BMI $(p=0.001)$, time of surgery $(p=0.029)$, prophylactic drainage $(p=0.006)$ and time under anesthesia $(p=0.012)$ to be associated to AL. Multivariate analysis showed BMI greater than $30 \mathrm{~kg} / \mathrm{m}^{2}$ ( $p=0.006$; OR 2.6 CI 1.3-5.2) and "after hours" construction of an anastomosis $(p=0.030$; OR 2.2 CI 1.1-4.5) to be independent risk factors.

Conclusion BMI greater than $30 \mathrm{~kg} / \mathrm{m}^{2}$ and "after hours" construction of an anastomosis were independent risk factors for colorectal anastomotic leakage.

Keywords After-hours - Colorectal surgery .

Anastomotic leakage $\cdot$ Body mass index

\section{Introduction}

Anastomotic leakage (AL) is the major complication after colorectal surgery. It is poorly understood as illustrated by the vast body of confirming and contradicting publications on virtually every known potential risk factor. The great variation of reported incidences of $\mathrm{AL}$, between $3 \%$ and $19 \%[1,2]$, illustrates this as well.

The poor understanding of AL may be due to its multifactorial aspect. In addition, studies on potential risk factors are performed in different populations throughout the world and, therefore, show many different independent risk factors for anastomotic leakage, varying from diverticular disease [3] and rectal resection [4] to weight loss [5], urgent operation [6], smoking [7] and BMI [8]. The factor "surgeon" is important as well since it is shown that leakage risk is lower when patients are operated upon by a high-volume surgeon [9]. 
The group of potential risk factors for AL that is studied in literature is generally quite similar and contains, in addition to the aforementioned factors, mechanical bowel preparation (MBP) [10], prophylactic drainage (PD) [11], ASA-score [4], prolonged operating time [4], use of corticosteroids [12], anastomotic configuration [13], hand-sutured vs. stapled anastomosis [14], neoadjuvant radiotherapy [15], laparoscopic vs. open surgery [16], and gender [8].

Despite the vast body of evidence on these potential risk factors AL remains poorly understood. Therefore, risk factor analysis should not be limited to these factors.

Since ischemia is considered to be one of the causes of AL, studying vascular disease, i.e., atherosclerosis, could be a new approach as suggested by Foster et al. [17]. Analysis of known risk factors for atherosclerosis like hypertension [18], dyslipidemia [19], smoking [20], diabetes mellitus [21] could be an interesting addition to the group of potential risk factors.

More and more publications show the danger of afterhours medical activity. Gray et al. showed after-hours surgery to be related to higher complications rate [22]. Fechner et al. showed that night time kidney transplantation enhances the risk for complications and graft failure [23]. In addition, several studies have shown that proficiency and situational awareness of physicians is less at night [24-27]. On the matter of colorectal surgery, the time of day at which the patient is operated has never been related to AL.

In short, risk factors for AL vary between different populations and, since it is a multifactorial problem, there are probably several unknown risk factors.

This means that more studies on this matter, from different hospitals throughout the world should be performed, published and compared in order to improve understanding of this complication.

This study aims to describe the population of patients with primary colorectal anastomosis treated in a Dutch tertiary referral center and to determine the incidence and risk factors of $\mathrm{AL}$ in this population.

\section{Materials and methods}

All patients who have undergone surgery on the colon or rectum in the period 1997-2007 were selected in the electronic archive by means of operation code of the relevant operations. In this population, patients whom received a primary anastomosis, involving colon and or rectum, were selected. The electronic- and paper patient records were used to score patient-, surgery-, and disease-related factors for risk factor analysis and description of the patient population.

The patient-related factors analyzed in this study were body mass index (BMI), age, gender, diabetes mellitus (DM), smoking, cardiac- and pulmonary comorbidity, history of vascular disease, ASA-score, use of steroids, statins, and anti-hypertensive medication (Table 1).

The surgery-related factors analyzed in this study were prophylactic drainage (PD), time of surgery, type of operation, anastomotic configuration, blood transfusion, surgeon vs. assistant, mechanical bowel preparation (MBP), laparoscopic vs. open operation, duration of surgery, stapled vs. hand-sutured anastomosis, urgent vs. elective operation (Tables 1 and 2). Operations were considered urgent when it was indicated in anaesthesiologists' records and the patient's file. Examples of included surgical emergencies were mechanical obstruction due to colorectal cancer and perforated diverticulitis.

The disease-related factors analyzed in this study were the type of neoadjuvant therapy, radio- or chemotherapy or a combination, surgical indication (Table 1).

In addition, several outcome parameters like wound infection, pneumonia, urinary tract infection, abdominal wound dehiscence, hospital stay and stay on the intensive care unit were analyzed as well

The primary outcome measure was clinically manifest anastomotic leakage, confirmed by imaging or relaparotomy. In case of imaging, leakage was considered to be present when free air or contrast was visible around the anastomosis. In case of relaparotomy, leakage was considered to be present when a dehiscent anastomosis was visualized.

Results are summarized as means and standard deviations or medians and ranges for the continuous variables; the categorical variables are summarized in frequencies. Median and mean values were compared between groups with and without AL by means of the Mann-Whitney test or Chi-square test in univariate analyses. Multivariate analysis was performed with factors that were significant in univariate analysis and consisted of multiple logistic regression with backwards elimination. In the final model, multiple logistic regression with backwards elimination with all factors, significant or nonsignificant in univariate analysis, was performed.

Operation duration was transformed logarithmically in this analysis to reduce the influence of outlying observations. A $p$ value of 0.05 (two-sided) was considered the limit of significance. Analyses were performed with "SPSS 15.0 " statistical software.

\section{Results}

Patient population

A total of 739 patients received a primary colorectal anastomosis over the selected period. This number included 90 ileocecal resections, 27 transverse colon resections, 250 right hemicolectomies, 30 subtotal colectomies, 64 left hemicolectomies, 155 sigmoid resections, 18 rectosigmoid 
Table 1 Description of operated population and univariate analysis

Population description and univariate analysis

\begin{tabular}{|c|c|c|c|c|}
\hline \multicolumn{2}{|l|}{ Factor } & \multirow{2}{*}{$\begin{array}{l}\text { Anastomotic leakage } \\
33(9 \%)\end{array}$} & \multirow{2}{*}{$\begin{array}{l}\text { No anastomotic leakage } \\
343(91 \%)\end{array}$} & \multirow{2}{*}{$\frac{p \text { value }}{0.001}$} \\
\hline BMI $\left(\mathrm{kg} / \mathrm{m}^{2}\right)^{\mathrm{a}}$ & $<25$ & & & \\
\hline & $25-30$ & $13(6 \%)$ & $217(94 \%)$ & \\
\hline & $>30$ & $15(20 \%)$ & $62(81 \%)$ & \\
\hline \multicolumn{2}{|l|}{ Age (years) ${ }^{b}$} & $61(22-84)$ & $60(18-99)$ & 0.953 \\
\hline \multicolumn{2}{|l|}{ Gender $(\mathrm{M} / \mathrm{F})$} & $32(9 \%) / 32(9 \%)$ & $346(91 \%) / 329(91 \%)$ & 0.951 \\
\hline \multicolumn{2}{|l|}{ Smoker ${ }^{\mathrm{c}}$} & Yes: $16(9 \%)$ No: $44(8 \%)$ & Yes: 163 (91\%) No: 485 (92\%) & 0.918 \\
\hline \multicolumn{2}{|l|}{ Use of steroids ${ }^{c}$} & Yes: $13(12 \%)$ No: $51(8 \%)$ & Yes: 96 (88\%) No: 569 (92\%) & 0.277 \\
\hline \multicolumn{2}{|c|}{$\begin{array}{l}\text { Use of Anti-hypertensive } \\
\text { medication }^{c}\end{array}$} & Yes: 7 (5\%) No: $55(9 \%)$ & Yes: 137 (95\%) No: 532 (91\%) & 0.116 \\
\hline \multicolumn{2}{|c|}{ Use of statines ${ }^{c}$} & Yes: 6 (9\%) No: $56(8 \%)$ & Yes: 63 (91\%) No: 608 (92\%) & 1.000 \\
\hline \multicolumn{2}{|l|}{ ASA-score ${ }^{c}$} & $\begin{array}{l}\text { I: } 13 \text { (20\%) II: } 27 \text { (42\%) III: } 20(31 \%) \\
\text { IV: } 1(2 \%) \text { V: } 0(0 \%)\end{array}$ & $\begin{array}{l}\text { I: } 162(24 \%) \text { II: } 303(45 \%) \text { III: } 125(19 \%) \\
\text { IV: } 20(3 \%) \text { V: } 1(0,1 \%)\end{array}$ & 0.203 \\
\hline \multicolumn{2}{|c|}{ History of vascular disease ${ }^{c}$} & Yes: $11(9 \%)$ No: $51(8 \%)$ & Yes: $106(91 \%)$ No: $564(92 \%)$ & 0.831 \\
\hline \multicolumn{2}{|c|}{ Cardiac comorbidity $^{\mathrm{c}}$} & Yes: $13(9 \%)$ No: $49(8 \%)$ & Yes: 134 (91\%) No: 539 (92\%) & 0.974 \\
\hline \multicolumn{2}{|c|}{ Pulmonary comorbidity ${ }^{\mathrm{c}}$} & Yes: 8 (9\%) No: $49(8 \%)$ & Yes: 85 (91\%) No: 563 (92\%) & 1.000 \\
\hline \multicolumn{2}{|c|}{ Diabetes mellitus ${ }^{\mathrm{c}}$} & Yes: $6(9 \%)$ No: $57(9 \%)$ & Yes: 60 (91\%) No: 606 (91\%) & 1.000 \\
\hline \multicolumn{2}{|l|}{ Blood transfusion $^{\mathrm{c}}$} & Yes: 14 (13\%) No: 48 (8\%) & Yes: 91 (87\%) No: 550 (92\%) & 0.114 \\
\hline \multicolumn{2}{|c|}{ Mechanical bowel preparation ${ }^{\mathrm{d}}$} & Yes: $37(8 \%)$ No: $16(10 \%)$ & Yes: 436 (92\%) No: $142(90 \%)$ & 0.460 \\
\hline \multicolumn{2}{|c|}{ Neoadjuvant radiotherapy } & Yes: 1 (8\%) No: 63 (9\%) & Yes: 11 (92\%) No: 664 (91\%) & 1.000 \\
\hline \multirow{2}{*}{\multicolumn{2}{|c|}{ Anastomotic configuration ${ }^{\mathrm{c}}$}} & E-E $7(12 \%)$ E-S $2(2 \%)$ & E-E 52 (88\%) E-S 90 (98\%) & 0.075 \\
\hline & & S-E $21(11 \%)$ S-S $33(9 \%)$ & S-E $170(89 \%)$ S-S 345 (91) & \\
\hline \multicolumn{2}{|l|}{ Surgical indication } & $\begin{array}{l}\text { Cancer: } 37 \text { (9\%) IBD: } 6 \text { (5\%) Diverticular } \\
\text { disease: } 3(5 \%) \text { Other: } 18(12 \%)\end{array}$ & $\begin{array}{l}\text { Diverticular disease: } 53 \text { (95\%) IBD: } 109(95 \%) \\
\text { Cancer: } 374 \text { (91\%) Other: } 139(89 \%)\end{array}$ & 0.249 \\
\hline \multirow{2}{*}{\multicolumn{2}{|c|}{ Approach }} & Laparotomy: $59(9 \%)$ & Laparotomy: 588 (91\%) & 0.328 \\
\hline & & Laparoscopy: 5 (5\%) & Laparoscopy: 87 (95\%) & \\
\hline \multicolumn{2}{|c|}{ Surgeon vs. assistant } & Surgeon 13 (10\%) Assistant 51 (8\%) & Surgeon 114 (90\%) Assistant 561 (92\%) & 0.603 \\
\hline \multicolumn{2}{|c|}{ Stapled vs. hand-sutured ${ }^{\mathrm{c}}$} & Stapled $5(8 \%)$ Sutured $57(9 \%)$ & Stapled 57 (92\%) Sutured 617 (91\%) & 1.000 \\
\hline \multicolumn{2}{|c|}{ Urgent vs. Elective } & Urgent: 19 (12\%) Elective: 45 (8\%) & Urgent: 137 (88\%) Elective: 538 (92\%) & 0.110 \\
\hline \multirow{2}{*}{$\begin{array}{l}\text { Prophylactic } \\
\text { drainage }\end{array}$} & Yes & $21(15 \%)$ & $121(85 \%)$ & 0.006 \\
\hline & No & $43(7 \%)$ & $554(93 \%)$ & \\
\hline \multirow[t]{2}{*}{ Time of surgery ${ }^{\mathrm{e}}$} & During-hours & $49(8 \%)$ & $584(92 \%)$ & 0.029 \\
\hline & After-hours & $15(14 \%)$ & $91(86 \%)$ & \\
\hline \multicolumn{2}{|c|}{ Duration of operation (minutes) ${ }^{\mathrm{b}}$} & $163(70-365)$ & $144(49-497)$ & 0.038 \\
\hline \multicolumn{2}{|c|}{ Time under anesthesia (minutes) ${ }^{b}$} & $226(110-426)$ & $195(75-630)$ & 0.012 \\
\hline
\end{tabular}

$B M I$ Body mass index $\left(\mathrm{kg} / \mathrm{m}^{2}\right), S D$ standard deviation, $A S A$ American Society of Anesthesiologists

${ }^{\mathrm{a}}$ BMI missing $47(6,4 \%)$

${ }^{\mathrm{b}}$ Expressed in median and range

${ }^{\mathrm{c}}$ Data do not add up to 739 due to occasional missing of data

${ }^{\mathrm{d}}$ MBP unknown in $108(14,5 \%)$ patients

${ }^{\mathrm{e}}$ During-hours is defined as the hours in which a new elective procedure was allowed to start (i.e. between $7.45 \mathrm{~h}$ and $15.30 \mathrm{~h}$ ). After-hours is defined as the period in which patients were operated upon by the operating team that is on call (i.e. between $15.30 \mathrm{~h}$ and $7.45 \mathrm{~h}$ ).

${ }^{\mathrm{f}}$ The group with nine non-traditional resections ("other" in Table 2) was excluded for risk factor analysis

resections and 96 low anterior resections after the total mesenterial excision principle (TME). Nine patients underwent non-traditional resections, consisting of eight anastomosis without resection when intraoperatively, the tumor appeared to have progressed to far and one resection of transverse and descending colon. These nine patients were excluded for further risk factor analysis. A total of 92 (13\%) operations were performed laparoscopically, of which $38(41 \%)$ were converted. The population is described in Table 1. 
Table 2 The incidence of anastomotic leakage stratified for the performed operations

"Other" contained eight anastomoses without resection when, intraoperatively, the tumor appeared to have progressed to far and one resection of transverse and descending colon

Analysis of the anastomotic leakage rate per operation showed a significant trend $(p=$ 0.019 ), regarding the expected increase in leakage rate going from top to bottom in the table (the group "other" was not included in this calculation).
Performed operations

\begin{tabular}{lcrr}
\hline Procedure & No anastomotic leakage & Anastomotic leakage & Total \\
\hline (Neo)-ileocecal resection & $86(96 \%)$ & $4(4 \%)$ & 90 \\
Resection transverse colon & $23(85 \%)$ & $4(15 \%)$ & 27 \\
(Extended) Hemicolectomy right & $235(94 \%)$ & $15(6 \%)$ & 250 \\
Subtotal colectomy & $26(87 \%)$ & $4(13 \%)$ & 30 \\
Hemicolectomy left & $56(88 \%)$ & $8(12 \%)$ & 64 \\
Resection sigmoid & $142(92 \%)$ & $13(8 \%)$ & 155 \\
Rectosigmoid resection & $15(83 \%)$ & $3(17 \%)$ & 18 \\
LAR/TME & $83(87 \%)$ & $13(13 \%)$ & 96 \\
Other & $9(100 \%)$ & $0(0 \%)$ & 9 \\
Total & $675(91.3 \%)$ & $64(8.7 \%)$ & 739 \\
\hline
\end{tabular}

Overall, the median duration of anesthesia was $217 \mathrm{~min}$ (range 75-630 $\mathrm{min}$ ) and the median duration of operation was $162 \mathrm{~min}$ (range $49-497 \mathrm{~min}$ ). A total of $633(86 \%)$ patients were operated upon during-hours, which was defined as the hours in which a new elective procedure was allowed to start (i.e., between $7.45 \mathrm{~h}$ and $15.30 \mathrm{~h}$ ). This means that generally, the elective program ended around $17.30 \mathrm{~h}-18.00 \mathrm{~h}$. A total of 106 patients (14\%) were operated upon after-hours, which was defined as the period in which patients were operated upon by the operating team that is on call (i.e., between $15.30 \mathrm{~h}$ and $7.45 \mathrm{~h}$ ).

A total of $156(21 \%)$ patients were operated upon in an emergency setting, 583 (79\%) patients were operated upon in elective setting. The median hospital stay after the operation was 13 days (range 1-180 days) and the median stay on ICU was 0 days (range $0-72$ days). The overall in hospital mortality rate was $4.7 \%$.

Incidence of anastomotic leakage

Anastomotic leakage was diagnosed in $64(8.7 \%)$ patients, of which nine patients (14\%) died due to consequences of anastomotic leakage (AL).

As depicted in Table 2, the highest incidence of $\mathrm{AL}$ occurred after resection of the rectosigmoid (17\%), followed by resection of the transverse colon $(15 \%)$ and low anterior resection $(13 \%)$. The median interval between operation and diagnosis of anastomotic leakage was 8 days (range 2-61 days).

\section{Risk factor analysis}

Analysis of the anastomotic leakage rate per operation showed a significant trend, indicating that higher leakage rates occurred as the anastomosis was constructed further downstream ( $p=0.019$; Table 2). Other potential risk factors for anastomotic leakage that reached significance in the univariate analysis were BMI $(p=0.001)$, PD $(p=0.006)$, time of surgery $(p=0.029)$, duration of operation and anesthesia $(p=0.038, p=0.012$; Table 1$)$.

Multivariate analysis showed body mass index (BMI) and time of surgery, classified in "during-" and "after hours", to be independent risk factors (Table 3). Prophylactic drainage was significantly associated with anastomotic leakage as well (Table 3).

Adjusted for these factors, no relation was found between leakage rate and the level of anastomosis.

Patient outcome

Mortality rate amongst the patients with anastomotic leakage (14.1\%) was significantly higher than amongst the

Table 3 Significant results of multivariate analysis

\begin{tabular}{lllll}
\hline Multivariate analysis & & & & \\
\hline Factor & & OR & $95 \%$ CI & $p$ value \\
\hline BMI $\left(\mathrm{kg} / \mathrm{m}^{2}\right)$ & $<25$ & 1 & - & - \\
& $25-30$ & 0.6 & $0.3-1.14$ & 0.115 \\
& $>30$ & 2.6 & $1.3-5.2$ & 0.006 \\
Time of surgery & During-hours & & \\
& After-hours & 2.2 & $1.1-4.5$ & 0.030 \\
Prophylactic drainage & No & 1 & - & - \\
& Yes & 2.8 & $1.5-5.1$ & 0.001 \\
\hline
\end{tabular}

The group "other" was excluded for the risk factor analysis; therefore, the total number of patients included is 730 . Reference categories are indicated by $\mathrm{OR}=1$

OR Odds Ratio, CI Confidence interval

${ }^{a}$ Time of surgery is defined as the hours in which a new elective procedure was allowed to start (i.e. between $7.45 \mathrm{~h}$ and $15.30 \mathrm{~h}$ ). After-hours is defined as the period in which patients were operated upon by the operating team that is on call (i.e., between $15.30 \mathrm{~h}$ and $7.45 \mathrm{~h})$. 
patients without $\mathrm{AL}(3.9 \% ; p=0.001)$. The incidence of urinary tract infections, pneumonia, ileus, and wound infections did not differ significantly. Abdominal wound dehiscence (AWD), however, occurred more often in the group of patients with AL ( $8 \%$ vs. $5 \%, p=0.046)$.

The mean hospital stay after operation and the stay at the ICU of patients with AL were 47 days and 7 days, respectively, whereas patients without AL stayed in the hospital 14 days and 1 days at the ICU, respectively $(p=0.000)$.

\section{Discussion}

Leakage of a colorectal anastomosis still is a vast problem despite decades of research on this matter. This study was performed in order to improve understanding of this complication and to study new potential risk factors.

Incidence of anastomotic leakage

In this study, an overall incidence of $8.7 \%$ was found over a period of 10 years. In literature leakage rates between 3\% and $6 \%$ are reported for colonic anastomosis [28, 29], while leakage rates between $16 \%$ and $19 \%$ for low colorectal anastomosis are reported $[2,30]$. Therefore this finding can be considered "average" considering anastomoses in the colon as well as in the rectum were included. The trend that was found in our study, i.e., higher leakage rates with lower anastomosis, is well-known too [31].

The leakage rate after resection of the transverse colon (four of 27) is remarkably high, which is not confirmed in literature [6]. Possibly the involvement of the watershed area at the splenic flexure and Griffiths' critical point (insufficient marginal artery at splenic flexure) contribute to this high leakage rate [32]. However, since this group is relatively small and the $95 \%$ confidence intervals for the leakage rate is wide (4-34\%), this finding should be considered only as an indication for further research.

\section{Risk factor analysis}

The multivariate risk factor analysis showed BMI, time of operation and prophylactic drainage to be major factors, independently associated with anastomotic leakage.

A BMI higher than 30, which is considered obese according to the Centers of Disease Control and Prevention (CDC) [33], increased the risk for developing leakage almost three-fold. As to the explanation of this correlation, it remains unclear whether obesity indicates a defect of tissue structure and healing, whether the increased intraabdominal pressure plays a role or whether construction of an anastomosis simply is technically more demanding because of thick mesenteries and epiploic appendices. A possible solution might be to lower the threshold for construction of a diverting stoma in this population [31]. However, since construction of a stoma is difficult in patients with high BMI, sensibilization and a higher alert for anastomotic leakage in these patients on the ward is a more logical consequence of this finding.

Patients operated upon after-hours had more than a twofold increased risk of anastomotic leakage. This finding was independent of the urgency of the operation, which may be explained by the fact that urgent operations were also performed during-hours and elective operations were performed after-hours (Table 4). The latter occurs regularly due to incoming trauma- or transplantation patients that require immediate operation (Table 4 ).

Possible explanations could be found in the fact that significantly more anastomoses were constructed by residents after-hours $(95 \%)$ than during-hours $(81 \% ; p<0.001)$ and that after-hours, obviously, significantly more urgent operations were performed $(86 \%)$ than during-hours $(10 \%$; $p<0.001)$. However, these factors were no independent risk factors in multivariate analysis.

Since many potential confounders have been accounted for and are not significant (Table 1), it is reasonable to assume a decreased technical performance of the operating team has contributed to a higher leakage rate at night. This assumption is supported by several studies that have shown medical errors to occur more often at night [24, 34]. In addition, physicians appear to be less proficient at night, which leads to more errors at night than during daytime [25-27].

Decreased non-technical skills of the operating team at night, like teamwork- and management skills and situational awareness, could have contributed to higher leakage rates as well. Situational awareness (SA), defined as the ability of the surgeon to observe, understand and predict events in the operating room (OR), appears to be closely related to technical error rates [35]. In addition, the situational awareness, teamwork- and management skills of the anesthetists and nurses may have an important impact on the outcome of surgical patients as well [27]. Based upon these results, it should be considered to construct a diverting stoma when after-hours construction of a colorectal anastomosis is required.

Table 4 Distribution of urgent and elective operations

Urgent vs. Elective

\begin{tabular}{llr}
\hline Time of surgery & Urgent & \multicolumn{1}{c}{ Elective } \\
\hline During hours & $64(10 \%)$ & $569(90 \%)$ \\
After-hours & $92(87 \%)$ & $14(13 \%)$ \\
\hline
\end{tabular}


Most studies addressing the problem of anastomotic leakage focus on the urgency of the operation. However, few studies report on the time of surgery in relationship to leakage rate. Higher leakage rates at night may be caused by a decreased technical and/or non-technical performance of the entire operating team. Given the current focus of society on medical errors and its prevention, more research should be done on this aspect of colorectal surgery.

Prophylactic drainage (PD) appeared to be associated with anastomotic leakage, suggesting that it is a risk factor. However, statistical analysis shows that significantly more drains are placed after LAR ( $54 \%$ of all drains; $p=0,001)$ and that the duration of surgery on average is 70 min longer in the group that received a drain $(219 \mathrm{~min})$ than in the group without ( $148 \mathrm{~min} ; p=0,001$ ). The latter is most likely caused by difficulty of operation and adverse events occurring during operation. This shows that the significant association between drainage and anastomotic leakage reflects the prophylactic drain-policy in our hospital. Additionally, since no significant differences in leakage rates between groups with and without drainage were reported in literature [11], PD cannot be considered as a risk factor for anastomotic leakage.

\section{Patient outcome}

Mortality rate due to leakage was $14.1 \%$, which is comparable to mortality rates reported in literature [4, 29].

The aforementioned 33-day increase in mean hospital stay in case of anastomotic leakage was to be expected and is indicative for the enormous increase in costs that is accompanied by this complication.

Concerning the postoperative complications, abdominal wound dehiscence (AWD) occurred significantly more often in patients with anastomotic leakage. In literature, several factors associated with abdominal wound dehiscence, being advanced age, obesity, diabetes mellitus, and steroid use [36], are reported. In this population BMI ( $p=$ $0.06)$, presence of diabetes mellitus $(p=0.99)$ and use of steroids $(p=0.07)$ were not associated with AWD. The average age of surgery in the group without AWD was 57 years and in the group with AWD 67 years $(p=0.010)$. However, since age is not a factor associated with anastomotic leakage this finding cannot explain the correlation between AWD and leakage. Another possible explanation may be found in connective tissue disorders. To date, a substantial body of evidence exists correlating aortic aneurysms to incisional hernia, both caused by connective tissue disorders [37]. In addition, Stumpf et al. have shown colorectal anastomotic leakage is associated with disturbances of the extracellular matrix [38]. These findings suggest that a patient population exists that has a higher risk for developing aortic aneurysms, hernia and anastomotic leakage. In addition, this concept may contribute to the previously mentioned misunderstanding and lack of consensus on the matter of anastomotic leakage.

\section{Conclusion}

Anastomotic leakage after colorectal surgery remains a major complication. In our hospital, overall leakage rate is $8.7 \%$ and mortality rate is $14.1 \%$ in patients with anastomotic leakage. BMI greater than $30 \mathrm{~kg} / \mathrm{m}^{2}$ and "after hours" construction of the anastomosis were independent risk factors for anastomotic leakage.

Open Access This article is distributed under the terms of the Creative Commons Attribution Noncommercial License which permits any noncommercial use, distribution, and reproduction in any medium, provided the original author(s) and source are credited.

\section{References}

1. Hyman N et al (2007) Anastomotic leaks after intestinal anastomosis: it's later than you think. Ann Surg 245(2):254-258

2. Matthiessen P et al (2007) Defunctioning stoma reduces symptomatic anastomotic leakage after low anterior resection of the rectum for cancer: a randomized multicenter trial. Ann Surg 246 (2):207-214

3. Platell C et al (2007) The incidence of anastomotic leaks in patients undergoing colorectal surgery. Colorectal Dis 9(1):71-79

4. Buchs NC et al (2008) Incidence, consequences, and risk factors for anastomotic dehiscence after colorectal surgery: a prospective monocentric study. Int J Colorectal Dis 23(3):265-270

5. Veyrie $\mathrm{N}$ et al (2007) Anastomotic leakage after elective right versus left colectomy for cancer: prevalence and independent risk factors. J Am Coll Surg 205(6):785-793

6. Choi HK, Law WL, Ho JW (2006) Leakage after resection and intraperitoneal anastomosis for colorectal malignancy: analysis of risk factors. Dis Colon Rectum 49(11):1719-1725

7. Sorensen LT et al (1999) Smoking and alcohol abuse are major risk factors for anastomotic leakage in colorectal surgery. Br J Surg 86(7):927-931

8. Kirchhoff P, Dincler S, Buchmann P (2008) A multivariate analysis of potential risk factors for intra- and postoperative complications in 1,316 elective laparoscopic colorectal procedures. Ann Surg 248(2):259-265

9. Borowski DW et al (2007) Impact of surgeon volume and specialization on short-term outcomes in colorectal cancer surgery. Br J Surg 94(7):880-889

10. Contant CM et al (2007) Mechanical bowel preparation for elective colorectal surgery: a multicentre randomised trial. Lancet 370(9605):2112-2117

11. Jesus EC et al. Prophylactic anastomotic drainage for colorectal surgery. Cochrane Database Syst Rev 2004(4):CD002100

12. Tresallet C et al (2008) Effect of systemic corticosteroids on elective left-sided colorectal resection with colorectal anastomosis. Am J Surg 195(4):447-451

13. Simillis $\mathrm{C}$ et al (2007) A meta-analysis comparing conventional endto-end anastomosis vs. other anastomotic configurations after resection in Crohn's disease. Dis Colon Rectum 50(10):1674-1687 
14. Fingerhut A et al (1994) Infraperitoneal colorectal anastomosis: hand-sewn versus circular staples. A controlled clinical trial. French Associations for Surgical Research. Surgery 116(3):484490

15. Peeters $\mathrm{KC}$ et al (2005) Risk factors for anastomotic failure after total mesorectal excision of rectal cancer. Br J Surg 92(2):211-216

16. Lourenco $\mathrm{T}$ et al (2008) Laparoscopic surgery for colorectal cancer: safe and effective?-a systematic review. Surg Endosc 22:1146-1160

17. Foster ME et al (1985) Colonic ischaemia and anastomotic healing. Eur Surg Res 17(3):133-139

18. Hansson L et al (1998) Effects of intensive blood-pressure lowering and low-dose aspirin in patients with hypertension: principal results of the Hypertension Optimal Treatment (HOT) randomised trial. HOT Study Group. Lancet 351(9118):17551762

19. Wadwa RP (2006) Cardiovascular disease risk in youth with diabetes mellitus. Rev Endocr Metab Disord 7(3):197-204

20. Zieske AW et al (2005) Smoking is associated with advanced coronary atherosclerosis in youth. Atherosclerosis 180(1):8792

21. Stamler J et al (1993) Diabetes, other risk factors, and 12-yr cardiovascular mortality for men screened in the multiple risk factor intervention trial. Diabetes Care 16(2):434-444

22. Gray A (2000) United Kingdom national confidential enquiry into perioperative deaths. Minerva Anestesiol 66(5):288-292

23. Fechner G et al (2008) Kidney's nightshift, kidney's nightmare? Comparison of daylight and nighttime kidney transplantation: impact on complications and graft survival. Transplant Proc 40 (5):1341-1344

24. Peberdy MA et al (2008) Survival from in-hospital cardiac arrest during nights and weekends. JAMA 299(7):785-792

25. Smith-Coggins R et al (1994) Relationship of day versus night sleep to physician performance and mood. Ann Emerg Med 24 (5):928-934
26. Rollinson DC et al (2003) The effects of consecutive night shifts on neuropsychological performance of interns in the emergency department: a pilot study. Ann Emerg Med 41(3):400-406

27. Catchpole $\mathrm{K}$ et al (2008) Teamwork and error in the operating room: analysis of skills and roles. Ann Surg 247(4):699-706

28. Mann B, Kleinschmidt S, Stremmel W (1996) Prospective study of hand-sutured anastomosis after colorectal resection. Br J Surg 83(1):29-31

29. Alves A et al (2002) Factors associated with clinically significant anastomotic leakage after large bowel resection: multivariate analysis of 707 patients. World J Surg 26(4):499-502

30. Pakkastie TE, Luukkonen PE, Jarvinen HJ (1995) Anterior resection controls cancer of the rectum as well as abdominoperineal excision. Eur J Surg 161(11):833-839

31. Rullier E et al (1998) Risk factors for anastomotic leakage after resection of rectal cancer. Br J Surg 85(3):355-358

32. Lange JF et al (2007) Riolan's arch: confusing, misnomer, and obsolete. A literature survey of the connection(s) between the superior and inferior mesenteric arteries. Am J Surg 193(6):742-748

33. (CDC), C.o.D.C.a.P. Centers of Disease Control and Prevention (CDC). [Website] 2008 [cited 2008 June 5th, 2008]

34. Neuberger JS et al (1984) Incidence of needlestick injuries in hospital personnel: implications for prevention. Am J Infect Control 12(3):171-176

35. Mishra A et al (2008) The influence of non-technical performance on technical outcome in laparoscopic cholecystectomy. Surg Endosc 22(1):68-73

36. Riou JP, Cohen JR, Johnson H Jr (1992) Factors influencing wound dehiscence. Am J Surg 163(3):324-330

37. Fassiadis $\mathrm{N}$ et al (2005) Randomized clinical trial of vertical or transverse laparotomy for abdominal aortic aneurysm repair. Br J Surg 92(10):1208-1211

38. Stumpf $M$ et al (2005) Changes of the extracellular matrix as a risk factor for anastomotic leakage after large bowel surgery. Surgery 137(2):229-234 